\title{
IDENTIFICACIÓN DE FACTORES MOTIVANTES DE COMPRA EN TIENDAS UNIVERSITARIAS: EL CASO DE LA UNIVERSIDAD NACIONAL DE COLOMBIA - SEDE MEDELLÍN
}

\author{
Identification of factors in university purchasing motivating shops: the case of the Universidad Nacional \\ de Colombia - sede Medellin
}

\author{
Cristian Oswaldo Ramírez-Villamil \\ Ingeniero Industrial. Universidad Nacional de Colombia. Medellín, Colombia. \\ cramirezvi@unal.edu.co \\ Katherin Lizeth Puerta-Meza \\ Ingeniera Industrial. Universidad Nacional de Colombia. Medellín, Colombia. \\ klpuertame@unal.edu.co
}

\section{Cómo citar / How to cite}

Ramírez-Villamil, C. y Puerta-Meza, K. (2016). Identificación de factores motivantes de compra en tiendas universitarias: el caso de la Universidad Nacional de Colombia- sede Medellín. Revista CEA, 2(4), 89-99.

Recibido: 27 de julio de 2015

Aceptado: 15 de marzo de 2016

\section{Resumen}

La identificación con la marca como factor de decisión y la exaltación del sentido de pertenencia, como efecto, son conceptos que se encuentran estrechamente relacionados cuando se analizan las decisiones de compra de artículos de la tienda universitaria, por parte de los estudiantes universitarios. En la presente investigación se indagó sobre los factores que motivan la compra de artículos universitarios por parte de los estudiantes de pregrado en la Universidad Nacional de Colombia Sede Medellín. Se logró identificar un factor significativo y ampliamente aceptado por los estudiantes encuestados, y presente en investigaciones realizadas en otras instituciones de diferentes países, correspondiente a la exaltación del sentido de pertenencia hacia la institución, aspecto que se ve reflejado directamente en el deseo de adquirir artículos que permitan dar a conocer su vínculo con la institución.
Palabras claves: tienda universitaria, identidad social, identificación con la marca, lealtad a la marca.

\begin{abstract}
The identification with the brand, as a decisive factor when making decisions and the exaltation of the sense of belonging, as effect, are concepts that are closely related when university students consider purchasing at the campus stores. The present study investigated the reasons that motivate university students to buy, particularly at the Universidad Nacional de Colombia, Medellin, where several factors influencing the decision to purchase certain items were identified. Moreover it was possible to confirm that a significant and widely accepted feature considered by students surveyed is the exaltation of the sense of belonging to the institution with the purchase of items related to it.
\end{abstract}

Keywords: university store, social identity, identification with the brand, brand loyalty. 


\section{INTRODUCCIÓN}

Partiendo del hecho de que el ser humano expresa a través de símbolos (logotipos, emblemas, marcas, figuras) sus deseos, sentimientos y emociones, se piensa en la manera como se pueden aprovechar este tipo de expresiones para fortalecer, en este caso, el sentido de pertenencia de los estudiantes hacia la Universidad Nacional de Colombia -sede Medellín- y reafirmar los valores institucionales, e igualmente hacer de la imagen institucional una marca, convirtiéndose en uno de los componentes para ampliar su difusión en el contexto regional, nacional e internacional, y lograr así su difusión. Por lo anterior, el objetivo de esta investigación es indagar sobre los factores que motivan la compra de artículos universitarios por parte de los estudiantes de pregrado en la Universidad Nacional de Colombia, sede Medellín.

Dado que se trabajó sobre el supuesto de la futura puesta en marcha de una tienda universitaria, que se ha denominado para efectos prácticos como tienda UN, se realizó un proceso de investigación exploratoria, a través de un diseño metodológico cuantitativo estructurado mediante la recolección de encuestas, con el fin de conocer los factores que podrían influir en las decisiones de los futuros compradores.

Entre los resultados esperados de este artículo se busca aportar una caracterización adecuada que permita identificar los productos que se ofrecerán a la comunidad universitaria y la forma como serán promocionados los productos de la tienda universitaria.

\section{REVISIÓN DE LITERATURA}

Al hablar de tiendas universitarias es necesario hacer un análisis de la situación en Colombia versus otros países, donde se puede apreciar que, en países como Estados Unidos, España o Brasil, se cuenta con tiendas universitarias que tienen un amplio recorrido, una estructura administrativa claramente definida y un gran reconocimiento, no solo al interior de las universidades sino por parte de público externo. Para el caso de Colombia, se pudo evidenciar claramente el rezago que existe en el país en cuanto a este tema; aunque es posible encontrar casos exitosos de algunas tiendas universitarias sobresalientes, como la tienda de la Universidad de Antioquia, la cual cuenta con alto reconocimiento y variedad de productos (Serrano \& Goyeneche, 2008).

El objetivo de las tiendas universitarias se centra en el fomento del sentido de pertenencia por parte de estudiantes y el reconocimiento por parte de una comunidad. Se tornó, por lo tanto, oportuno analizar la situación de la Universidad Nacional de Colombia, que cuenta con gran reconocimiento a nivel nacional, en especial la sede Medellín, donde se ha evidenciado la necesidad de un amplio sector de la comunidad universitaria por tener la posibilidad de adquirir artículos relacionados con la universidad a la cual pertenecen.

Este estudio toma relevancia debido a la limitada existencia de estudios académicos que relacionan la afiliación a una institución universitaria, y cómo la toma de decisiones frente a la compra de artículos licenciados por la institución se lleva a cabo (Phillips, Roundtree y Kim, 2014).

A pesar de conocerse poco sobre los factores reales que motivan la compra de artículos universitarios oficiales, uno de los más influyentes es el deseo de apoyar a la universidad en aspectos que estén más relacionados con la vida cotidiana, como lo puede ser la posesión de alguna prenda de vestir con el logo de la universidad. Lo anterior, no implica que los clientes sean solamente estudiantes o personas vinculadas a la universidad, ya que se ha identificado un público, menor en tamaño, en comparación con los afiliados a la institución, quienes portan artículos alusivos a una universidad por su reconocimiento a nivel regional $y / o$ nacional (Phillips, Roundtree y Kim, 2014), como es el 
caso de la Universidad Nacional de Colombia, al ser la más grande e importante del país.

En una investigación que se realizó con estudiantes de una universidad de Estados Unidos, se evidenció la tendencia de las personas afiliadas directamente a la institución a hacer visible su orgullo y enaltecer su sentido de pertenencia a través de la adquisición de artículos que continuamente exhiben, como lo son implementos de estudio o prendas de vestir (Phillips, Roundtree y Kim, 2014).

Se puede también hablar de una identificación con la marca, que está relacionada con la identidad del consumidor, al ser un concepto de identificación social en donde el sujeto se identifica con un objeto, que en este caso corresponde a una marca determinada (universidad), lo cual tiene beneficios psicológicos para la persona como el fortalecimiento de la autoestima, involucrando comportamientos como: la lealtad, el crossbuying, up buying, el boca a boca, la voluntad a pagar un precio mayor y la resistencia a aceptar información negativa sobre la compañía (Rubio, Villaseñor y Oubiña, 2014).

Lo anterior también es afirmado en estudios realizados por Aspara (2009), donde se plantea que la necesidad de consumo de todos los individuos y sus comportamientos de inversión están vinculados a unas psicologías relacionadas con la influencia, ya sea por apoyo o por lealtad a la marca.

Otro factor que se debe abordar es la lealtad, la cual es una cualidad del valor de marca, donde el consumidor escoge qué comprar de forma racional o irracional, pero en sí, generando una imagen positiva hacia la marca elegida (Peláez y Macía, 2010 citando a Delgado, 2004). Para Dick y Basu (1994) existen cuatro niveles de lealtad: lealtad verdadera (compromiso alto y recompra alta), lealtad latente (compromiso alto $y$ recompra baja), lealtad fingida (compromiso bajo y recompra alta) y la lealtad inexistente (compromiso y recompra bajos) (Ver Tabla 1).
La tienda universitaria, por su objeto de ser y por el escenario en el cual se desarrollará, debe generar lealtad verdadera, en el peor de los casos, lealtad latente, teniendo en cuenta que muchas de las personas vinculadas a la institución, no cuentan con altos recursos económicos.

Tabla 1. Modelo de lealtad de Dick y Basu

Tabla 1. Loyalty model from Dick \& Basu

\begin{tabular}{|c|c|c|c|}
\hline & \multicolumn{3}{|c|}{$\begin{array}{c}\text { Repetición del patrocinio } \\
\text { (Recompra) }\end{array}$} \\
\hline & & Alta & Baja \\
\hline \multirow{2}{*}{$\begin{array}{l}\text { Actitud } \\
\text { relativa } \\
\text { (compromiso) }\end{array}$} & Alta & $\begin{array}{l}\text { Lealtad } \\
\text { Verdadera }\end{array}$ & $\begin{array}{l}\text { Lealtad } \\
\text { latente }\end{array}$ \\
\hline & Baja & $\begin{array}{l}\text { Lealtad } \\
\text { fingida }\end{array}$ & $\begin{array}{l}\text { Lealtad } \\
\text { inexistente }\end{array}$ \\
\hline
\end{tabular}

Fuente: Dick y Basu, 1994.

En cuanto a la situación en Colombia sobre tiendas universitarias, se tiene la siguiente: Universidad de Antioquia, Universidad Pontificia Javeriana (Bogotá), Universidad Pontificia Bolivariana (Medellín), Universidad La Salle (Bogotá), Universidad del Rosario (Bogotá), Universidad Piloto de Colombia (Bogotá), Universidad EAFIT (Medellín) y Universidad Autónoma de Bucaramanga (UNAB) (Serrano y Goyeneche, 2008).

Un ejemplo de tienda universitaria, con un enfoque tanto de fomento de cultura institucional, como de promoción de la imagen o marca, es la Universidad de Caldas, en Manizales, quienes plantean en su página web oficial lo siguiente: «La Tienda de la Universidad de Caldas es una estrategia de difusión y promoción de la marca de la universidad que busca generar recordación de la institución en sus diferentes públicos a través de la comercialización de souvenirs» (Universidad de Caldas, 2014).

La tienda universitaria, más que un ente comercial, como se ha mencionado, resulta ser una herramienta para reforzar el sentido de pertenencia universitaria, en el sentido de que permite, por ejemplo, reforzar los valores institucionales y servir de promoción de su 
marca. Ejemplos de este tipo de ente institucional es la tienda de la Universidad Pontificia Bolivariana, en Medellín:

La Tienda Universitaria es un espacio de interacción de la marca UPB con sus diferentes públicos. En su oferta de artículos para uso cotidiano con diseños innovadores se promueven valores Institucionales, se estimula el sentido de pertenencia de los estudiantes, egresados y empleados de la Universidad y se apoya la identidad corporativa de la UPB al proyectarla de forma dinámica y coherente (Universidad Pontificia Bolivariana, 2014).

En una investigación referente al establecimiento de una tienda universitaria, realizada en la Fundación Universitaria del Área Andina, «se identificó la necesidad de desarrollar un proyecto de investigación que determine las variables que permitan la viabilidad del proyecto, dentro de la propuesta virtual, como la primera etapa de construcción epistémica del proyecto» (Fundación Universitaria del área Andina, 2011).

Según un estudio realizado en la ciudad de Medellín, los consumidores en esta localidad se dejan llevar, en gran parte, por las tradiciones heredadas de la familia, generando lealtad de marca para un producto en específico (Peláez y Macía, 2010). Al generar en cierto grupo de personas el hábito de compra en la tienda universitaria, servirá de pilar para que la marca de la universidad sea recordada en el mercado, y así atraer posibles clientes, generando una tradición.

El insumo principal de esta investigación es el hecho de que en la comunidad universitaria se encuentra una identidad cultural colectiva, por lo que resulta necesario entender cómo esta pueda influir en el diseño de cualquier artículo que pretenda representarla. Para lograr este primer acercamiento, se deben conocer las consideraciones que se hacen durante el proceso de diseño de los artículos y con ayuda de esta investigación saber los tipos, características y precios de los artículos que se ofrezcan en la tienda UN.

Una parte importante del diseño de un artículo, en este caso del tipo souvenir, es el cómo su fabricación se puede vincular con la tradición, con la técnica o directamente con la cultura del lugar de donde procede. "Podemos decir que los souvenir van desde los que señalan simplemente "yo estuve en", hasta los que constituyen un testimonio de una experiencia un poco más profunda» (Petit y Charpentier, 2013, p. 145).

A partir de este referente se facilita decidir sobre en qué tipo de artículos se debe enfocar la investigación, y serán entonces los artículos que, más allá de recordar que se estuvo en la UN, son una muestra tangible de lo que es en sí la UN y los valores sociales que esta implica. Aunque será este el enfoque principal de la investigación, también se deben incluir los otros artículos que, aunque de menor simbolismo, logran identificar a la UN y que seguramente los futuros clientes van a querer adquirir, probablemente porque son objetos de mayor exhibición.

Con base en lo anterior y reiterando el hecho de que la información sobre el diseño de los productos será abordada de manera superficial para efectos de seleccionar qué productos se ofrecerán principalmente en la tienda UN, se debe proceder a identificar algunos aspectos, los más representativos, de la cultura interna de la Universidad Nacional de Colombia, sede Medellín. Se optó entonces por incluir en la investigación los artículos que siendo destacados por su valor cultural y representación de la vida universitaria también abarcan de manera general los sentimientos de los posibles clientes.

Otro aspecto a considerar es la influencia que la marca, el logo o la licencia de una marca tiene sobre un producto, lo cual incita la compra impulsiva de este en la medida en que el cliente tiene algún tipo de afiliación con la institución o, 
como en el caso puntual de la investigación realizada por Kwon y Armstrong entre 2002 y 2006, con un equipo deportivo. En dicha investigación, se observó que el diseño y el logo desempeñan un papel de importancia en la toma de decisiones de compra de artículos, en este caso deportivo (Kwon y Armstrong, 2006). Con este resultado y las demás observaciones realizadas, se evidenció el impacto que tienen los símbolos de aquello con lo que se tiene afiliación en la decisión de adquirir un artículo, y en especial, cómo esa influencia se presenta de manera tal que induce a realizar la compra de manera inmediata o sin considerar muchos aspectos que de otra manera se pondrían en consideración al realizar una compra.

\section{METODOLOGÍA DE INVESTIGACIÓN}

La investigación que se llevó a cabo es de tipo exploratorio-descriptivo con un enfoque cuantitativo, utilizando como instrumento un cuestionario auto-administrado. El público objetivo fueron los estudiantes de pregrado activos de la Universidad Nacional de Colombia sede Medellín. Se seleccionaron las encuestas como método de recolección de información con el propósito de recolectar información valiosa de un gran número de estudiantes que permitiera garantizar un análisis más profundo y significativo. Además, la encuesta es ampliamente empleada como instrumento de recolección de información, ya que permite recolectar datos de manera rápida y eficaz (Casas-Anguita, Repullo-Labrador y DonadoCampos, 2003).

Se aplicaron 315 encuestas a los estudiantes de la Universidad Nacional de Colombia -sede Medellín, a través de un muestreo no probabilístico por criterio. El total de las encuestas se aplicó durante los días comprendidos del 17 a 21 de noviembre de 2014, en las diferentes sedes de la Universidad Nacional de Colombia sede Medellín.

La recolección de información es una etapa fundamental en el proceso investigativo, ya que se deben recolectar los datos necesarios sobre los atributos y variables implicadas en el objeto de estudio (Echauri, Minami y Sandoval, 2014). Por lo anterior, el diseño de la encuesta incluyó principalmente preguntas en formato Escala de Likert, ya que esta escala es aditiva con un nivel ordinal, donde el interrogado plantea su nivel de acuerdo o de desacuerdo con cada afirmación relativa al asunto estudiado, teniendo la ventaja de que las alternativas de respuesta son fijas para todas las proposiciones y todas tienen designado un peso equivalente (Namakforoosh, 2000).

Las preguntas finales del cuestionario se seleccionaron a partir del resultado de la encuesta piloto realizada a 30 estudiantes pertenecientes a la población objetivo; adicional a este tipo de preguntas se incluyeron preguntas dicotómicas y de selección múltiple con única respuesta, que permitieran agrupar ciertas características de los encuestados (entre ellas estrato y edad).

Posteriormente, se digitaron y tabularon, considerando finalmente 299 encuestas válidas (95\% del total aplicado) luego de realizar filtros de calidad. El análisis de resultados se planteó desde una perspectiva descriptiva mediante el cruce de las variables de interés que sirvieran como insumo para conocer los principales factores motivantes de compra (precio, afiliación, uso, producto, promoción) en tiendas universitarias.

\section{ANÁLISIS DE RESULTADOS}

Para la realización del respectivo análisis con los datos recolectados es preciso contar con la definición de categorías que permitan contrastar, a su vez, dos o más factores seleccionados de las respuestas proporcionadas por los encuestados. En dichas categorías se establece una estructura que se distribuye primeramente con la explicación de la categoría, seguida de la respectiva tabla que permitirá respaldar las conclusiones con base a resultados significativos. 


\section{Precio}

El precio se constituye como una de las variables que podría afectar la decisión de compra de los productos a ofrecer en la tienda UN. Considerando la población de la Universidad Nacional de Colombia -sede
Medellín, respecto del estrato socioeconómico, se procedió a cruzar datos obtenidos del estrato al que pertenecen los encuestados con la opinión que dieron cuando se les consultó por los montos de dinero que estarían dispuestos a pagar (ver Tabla 1).

Tabla 2. Estrato vs. Valor dispuesto a pagar por ropa (\$)

Table 2. Stratum vs. Willing value top pay for clothes (\$)

\begin{tabular}{cccccc}
\hline $\begin{array}{c}\text { ESTRATO / } \\
\text { PAGARÍA }\end{array}$ & No le interesa & $24.990-39.990$ & $40.000-74.990$ & $75.000-99.990$ & $100.000-120.000$ \\
\hline $1-2$ & $7,59 \%$ & $35,44 \%$ & $27,85 \%$ & $20,25 \%$ & $8,86 \%$ \\
$3-4$ & $8,12 \%$ & $27,41 \%$ & $38,07 \%$ & $17,77 \%$ & $8,63 \%$ \\
$4-5$ & $13,64 \%$ & $9,09 \%$ & $36,36 \%$ & $27,27 \%$ & $13,64 \%$ \\
Total general & $8,39 \%$ & $28,19 \%$ & $35,23 \%$ & $19,13 \%$ & $9,06 \%$ \\
\hline \multicolumn{7}{c}{}
\end{tabular}

Al evaluar el estrato socioeconómico de los estudiantes encuestados, se pudo observar cierto comportamiento acorde con el estrato al cual pertenecen. Por ejemplo, para la ropa que contiene el rango de precios más alto, se observa que los estudiantes que pertenecen a estratos 1 y 2 prefieren artículos más económicos. Un dato importante es que los estudiantes encuestados pertenecientes a estratos 3 y 4 , que representan el $66 \%$ de los encuestados, están dispuestos a pagar entre 40.000 y 75.000 pesos por una prenda de vestir, lo cual representa un $38,1 \%$ de los encuestados que pertenecen al rango del estrato mencionado.

Como se puede observar en la Tabla 3, el grupo de los encuestados pertenecientes a los estratos 3 y 4 demuestran en su gran mayoría interés por los elementos de escritorio y continúan manteniendo una intención de pago intermedia, que comparado con este mismo tipo de productos de otras marcas que se encuentran en el mercado, sigue siendo inferior.

Tabla 3. Estrato vs. Elementos de escritorio (\$)

Table 3. Stratum vs. Willing value to pay for office implements (\$)

\begin{tabular}{cccccc}
\hline ESTRATO / & No le interesa & $3.000-4.990$ & $5.000-7.990$ & $8.000-9.990$ & $10.000-12.000$ \\
PAGARÍA & $3,80 \%$ & $43,04 \%$ & $41,77 \%$ & $6,33 \%$ & $5,06 \%$ \\
\hline $1-2$ & $5,58 \%$ & $32,99 \%$ & $33,50 \%$ & $16,24 \%$ & $11,68 \%$ \\
$3-4$ & $13,64 \%$ & $50,00 \%$ & $27,27 \%$ & $4,55 \%$ & $4,55 \%$ \\
$4-5$ & $5,70 \%$ & $36,91 \%$ & $35,23 \%$ & $12,75 \%$ & $9,40 \%$ \\
\hline
\end{tabular}

Fuente: elaboración propia a partir de resultados de encuesta.

En la Tabla 3 se aprecia que, de los estudiantes encuestados, la mayoría prefiere los precios intermedios, independiente del tipo de artículo del cual se esté hablando, a excepción de los dulces, donde claramente se ve una marcada preferencia por los precios bajos, lo cual se puede deber a un poco interés por parte de los estudiantes en este tipo de artículos.

También es importante mencionar que debido a que la mayoría de los encuestados pertenecen a estratos 3 y 4 , con un $66 \%$ del total, este grupo será el que defina las preferencias del total 
general, es decir, el valor seleccionado por todos los encuestados será cercano al del grupo o estrato en mención.

\section{Afiliación}

Como se sabe, la universidad es un entorno en el que confluyen múltiples actividades y grupos de personas que pueden generar cierta afiliación, sentido de pertenencia o afectos y que pueden aprovecharse para acercar más a quiénes participan de estos, por medio de los artículos que comercializaría la tienda universitaria.

En principio, se buscó conocer a qué grupo(s) extracurricular(es) pertenecían los encuestados y luego se les preguntó por su intención de comprar o no artículos alusivos a los grupos en los que participaban. De este enfoque se obtuvieron los siguientes resultados.

La Tabla 4 evidencia que de los encuestados que pertenecen a algún grupo extracurricular, que corresponde a alrededor del 35\% del total de encuestados, quienes menos intención de compra tienen hacia artículos relacionados con el grupo extracurricular al que pertenecen, son los afiliados a grupos académicos; mientras que los pertenecientes a grupos culturales presentan una mayor tendencia a desear artículos alusivos al grupo cultural al cual pertenecen.

Los resultados apoyan lo propuesto por Salman (2014), respecto a la influencia que tiene la pertenencia a un grupo deportivo o atlético en las decisiones de compras de estudiantes universitarios.

Tabla 4. Estrato vs. Dulces (\$)

Table 4. Stratum vs. Willing value to pay for candies (\$)

\begin{tabular}{cccccc}
\hline $\begin{array}{c}\text { ESTRATO / } \\
\text { PAGARÍA }\end{array}$ & No le interesa & $1.000-1.990$ & $2.000-2.490$ & $2.500-2.990$ & $3.000-4.000$ \\
\hline $1-2$ & $10,13 \%$ & $51,90 \%$ & $16,46 \%$ & $12,66 \%$ & $8,86 \%$ \\
$3-4$ & $15,74 \%$ & $36,55 \%$ & $27,92 \%$ & $13,71 \%$ & $6,09 \%$ \\
$4-5$ & $22,73 \%$ & $18,18 \%$ & $36,36 \%$ & $9,09 \%$ & $13,64 \%$ \\
Total general & $14,77 \%$ & $39,26 \%$ & $25,50 \%$ & $13,09 \%$ & $7,38 \%$ \\
\hline
\end{tabular}

Fuente: elaboración propia a partir de resultados de encuesta.

\section{Uso}

Cuando se pensó en la idea de una tienda universitaria resultó natural pensar en que muy probablemente los productos no solo serían adquiridos por o para miembros de la comunidad universitaria, por lo que se quiso saber con qué fin, o para qué tipo de usos se destinarían los artículos que los encuestados comprarían.

Al iniciar el análisis de este aspecto se partió de la hipótesis de que algunos artículos tendrían más demanda que otros dependiendo del uso para el que se fuesen a adquirir, por ejemplo, las prendas de vestir, sobre las que se pensó que serían adquiridas para el uso propio. Lo que se halló al respecto se observa en la Tabla 5.
Tabla 5. Compra de artículos alusivos al grupo extracurricular

Table 5. Purchase of products allusive to extracurricular group

\begin{tabular}{|c|c|c|}
\hline Grupo & No & Si \\
\hline Deportivo & $50,00 \%$ & $50,00 \%$ \\
\hline Cultural & $46,15 \%$ & $53,85 \%$ \\
\hline Académico & $62,86 \%$ & $37,14 \%$ \\
\hline Total general & $53,47 \%$ & $46,53 \%$ \\
\hline
\end{tabular}

En la Tabla 5 se comparó la opción de comprar artículos para el uso propio con la pregunta en la que se indicaba el nivel de preferencia de acuerdo con cada tipo de artículo. Inicialmente se tuvo la hipótesis de que cuando se prefería comprar artículos para el uso propio, estos por 
lo general serían mayormente prendas de vestir $y$ en algunos casos accesorios. Como bien se puede apreciar, más del $80 \%$ de las personas que indicaron que comprarían artículos para el uso propio tienen una tendencia importante a preferir la adquisición de prendas de vestir, y curiosamente accesorios.

\section{Producto}

En este punto se deben considerar los productos que se podrían ofrecer en la tienda universitaria, y que podrían tener buena acogida en la población objetivo. Como fue de esperar, se hizo necesario conocer los productos que se ofrecerían en la tienda UN, para de esta forma analizar qué tanta disposición tendrían los encuestados de pagar por esos productos.
En la Tabla 6, inicialmente se identifica que quienes dijeron no interesarles la adquisición de prendas de vestir muestran no estar dispuestos a pagar por dichos artículos, mostrando la coherencia interna entre las respuestas de los encuestados. Un dato curioso es que las personas que manifestaron poco interés por los accesorios se encuentran dispuestas a pagar por ellos, evidentemente un poco menos que las personas que manifestaron un mayor interés por los artículos en esta categoría.

Para el caso de los dulces se ven dos cosas importantes. La primera, que gran parte de las personas que dicen no interesarles, si pagaran por ellos no destinarían mucho dinero a estos, y la segunda es que incluso las personas que les interesaría mucho la dulcería tampoco pagarían mucho por estos productos.

Tabla 6. Uso propio vs. Articulo

Table 6. Personal use vs. Article

\begin{tabular}{cccccc}
\hline \multirow{2}{*}{ Uso Propio } & \multicolumn{5}{c}{ Preferencia } \\
\cline { 2 - 6 } & $\begin{array}{c}\text { No le } \\
\text { gustaría }\end{array}$ & Poco & Neutral & Algo & Mucho \\
\hline Prendas de Vestir & $4.05 \%$ & $7.29 \%$ & $17.00 \%$ & $29.15 \%$ & $42.51 \%$ \\
Accesorios & $4.42 \%$ & $9.64 \%$ & $14.46 \%$ & $20.08 \%$ & $51.41 \%$ \\
\hline \multicolumn{7}{r}{ Fuente: elaboración propia a partir de resultados de encuesta. }
\end{tabular}

Tabla 7. Interés en ropa vs Dinero dispuesto a pagar Table 7. Clothes interest vs. Willing value to pay for clothes

\begin{tabular}{lccccc}
\hline $\begin{array}{l}\text { INTERÉS } \\
\text { PAGARÍA }\end{array}$ & No le interesa & $24.990-39.990$ & $40.000-74.990$ & $75.000-99.990$ & $100.000-120.000$ \\
\hline No le gustaría & $58,33 \%$ & $25,00 \%$ & $0,00 \%$ & $0,00 \%$ & $16,67 \%$ \\
Poco & $21,05 \%$ & $26,32 \%$ & $36,84 \%$ & $15,79 \%$ & $0,00 \%$ \\
Neutral & $9,62 \%$ & $34,62 \%$ & $32,69 \%$ & $19,23 \%$ & $3,85 \%$ \\
Algo & $6,59 \%$ & $23,08 \%$ & $36,26 \%$ & $21,98 \%$ & $12,09 \%$ \\
Mucho & $1,64 \%$ & $29,51 \%$ & $39,34 \%$ & $19,67 \%$ & $9,84 \%$ \\
Total general & $8,11 \%$ & $28,04 \%$ & $35,47 \%$ & $19,26 \%$ & $9,12 \%$ \\
\hline
\end{tabular}

Fuente: elaboración propia a partir de resultados de encuesta.

\section{Promoción}

Otro aspecto a tener en cuenta dentro del análisis y que puede proporcionar información de utilidad es la promoción de la tienda, Al apreciar los resultados obtenidos en la realización de la encuesta, en cuanto a la haciendo referencia a la forma como prefieren los encuestados conocer la tienda y sus productos y qué relación puede guardar este aspecto con algún otro factor como el estrato, el interés en adquirir determinados productos, etc. importancia de encontrar información de determinadas formas, se puede observar en la 
Tabla 8, que el $62,42 \%$ de la población encuestada considera muy importante encontrar información de los productos de la tienda universitaria en la página web, $65,10 \%$ en las redes sociales y el 37,58\% alrededor del campus. Lo que da indicios de dónde se puede compartir la información de los productos de la tienda universitaria, ya que los estudiantes hacen uso frecuente del Internet y también están pendientes de la información que se expone alrededor del campus, aunque en menor medida, como carteleras de información o pendones llamativos alusivos a una publicidad determinada. En este caso se aprovecharía esto y se haría la publicidad necesaria para que la tienda UN tenga la acogida favorable esperada.

Tabla 8. Interés en dulcería vs Dinero dispuesto a pagar

Table 8. Candies interest vs. Willing value to pay

\begin{tabular}{cccccc}
\hline $\begin{array}{c}\text { INTERÉS / } \\
\text { PAGARÍA }\end{array}$ & No le interesa & $\mathbf{1 . 0 0 0 - 1 . 9 9 0}$ & $\mathbf{2 . 0 0 0 - 2 . 4 9 0}$ & $\mathbf{2 . 5 0 0 - 2 . 9 9 0}$ & $\mathbf{3 . 0 0 0 - 4 . 0 0 0}$ \\
\hline 1 & $22,00 \%$ & $44,00 \%$ & $30,00 \%$ & $0,00 \%$ & $4,00 \%$ \\
2 & $16,67 \%$ & $33,33 \%$ & $30,30 \%$ & $15,15 \%$ & $4,55 \%$ \\
3 & $20,63 \%$ & $34,92 \%$ & $28,57 \%$ & $9,52 \%$ & $6,35 \%$ \\
4 & $10,20 \%$ & $42,86 \%$ & $18,37 \%$ & $22,45 \%$ & $6,12 \%$ \\
5 & $4,48 \%$ & $41,79 \%$ & $20,90 \%$ & $17,91 \%$ & $14,93 \%$ \\
Total general & $14,58 \%$ & $38,98 \%$ & $25,76 \%$ & $13,22 \%$ & $7,46 \%$ \\
\hline \multicolumn{7}{c}{}
\end{tabular}

Tabla 9. Información de los productos

Table 9. Product information

\begin{tabular}{lccccc}
\hline & $\begin{array}{c}\text { Muy } \\
\text { importante }\end{array}$ & Importante & Normal & $\begin{array}{c}\text { Poco } \\
\text { importante }\end{array}$ & $\begin{array}{c}\text { Sin } \\
\text { importancia }\end{array}$ \\
\hline Página Web & $62,42 \%$ & $22,82 \%$ & $13,09 \%$ & $0,67 \%$ & $1,01 \%$ \\
Redes sociales & $65,10 \%$ & $18,79 \%$ & $14,43 \%$ & $0,67 \%$ & $1,01 \%$ \\
Alrededor del campus & $37,58 \%$ & $25,17 \%$ & $16,44 \%$ & $17,45 \%$ & $3,36 \%$ \\
\hline
\end{tabular}

Fuente: elaboración propia a partir de resultados de encuesta.

En cuanto a la pregunta 9, a la gente le gustaría tener información gráfica (fotografías) en la publicidad que se haga de la tienda UN y conocer las promociones y los precios de los artículos que la tienda ofrece, ya que estas opciones fueron las más frecuentes en la población encuestada. Frente a lo anterior, en la Tabla 10 se observa que la población encuestada de estratos 5-6 se muestra indiferente en cuanto al tipo de información que se exhiba de los productos; por el contrario, la población universitaria de estratos 3-4 es la que muestra mayor empatía con conocer el precio de los productos, lo que es muy importante, ya que el $66 \%$ de la población encuestada pertenece a estos estratos.

Tabla 10. Tipo de información deseada en la publicidad de la tienda UN

Table 10. Type of information wanted in advertising

\begin{tabular}{cc}
\hline Fotografías & $93,96 \%$ \\
Descripción & $73,15 \%$ \\
Precio & $96,31 \%$ \\
Disponibilidad & $63,76 \%$ \\
Promociones & $89,26 \%$ \\
\hline
\end{tabular}

Fuente: elaboración propia a partir de resultados de encuesta. 
Tabla 11. Estrato vs Conocer la promoción de los productos

Table 11. Stratum vs. Need to know discounts of products

\begin{tabular}{cccc}
\hline Estrato & No & $\mathrm{Si}$ & Total general \\
\hline 1 & $28,13 \%$ & $26,32 \%$ & $26,51 \%$ \\
2 & $65,63 \%$ & $66,17 \%$ & $66,11 \%$ \\
3 & $6,25 \%$ & $7,52 \%$ & $7,38 \%$ \\
\hline
\end{tabular}

Fuente: elaboración propia a partir de resultados de encuesta.

En la Tabla 11 se tiene que el estrato social con respecto a las promociones de los productos, siguen siendo más importantes para las personas de estratos 3 que para los estratos 1 y 2. Por el contrario, las personas de estratos 5 y 6 no tienen interés en conocer estas cosas. Es por ello que al tener un $66 \%$ de la población encuestada en los estratos 3-4, es de gran relevancia considerar los resultados de esta pregunta y la información que se puede obtener de ella, por lo que se debe pensar en un plan de publicidad y dar a conocer todo lo referente a los productos que se tendrán en la tienda UN, promociones, bonos, descuentos, obsequios y demás.

Además, por ser el campus de dos núcleos, se puede pensar en una publicidad no por medios masivos, sino por carteles, folletos y por Internet, aunque no se debe dejar de lado el «boca a boca», que por estar toda la comunidad universitaria en contacto diariamente puede llegar a ser muy efectiva.

\section{CONCLUSIONES}

La puesta en marcha de una tienda universitaria en la Universidad Nacional de Colombia - sede Medellín, resultaría viable desde el punto de vista de los clientes potenciales, es decir, los estudiantes, quienes manifestaron compromiso y aceptación hacia la idea de implementar una tienda UN, con una aceptación del 97\% frente a un $3 \%$ en desacuerdo. El compromiso se observa en la disposición por parte de los estudiantes a adquirir sus artículos como mecanismo para resaltar el sentido de pertenencia que se tiene hacia la universidad, puesto que esta última necesidad representa más del $50 \%$ de los factores que motivan a los encuestados a adquirir los productos.

Además, un $80 \%$ de los encuestados concuerdan al afirmar que contar con dichos artículos oficiales incrementaría el sentimiento de orgullo y el sentido de pertenencia hacia la institución, al poder exhibir artículos alusivos a la universidad. Esto reafirma el supuesto principal de esta investigación, es decir, que la principal motivación de este tipo de consumidores (estudiantes universitarios) sobre esta clase de productos licenciados por la universidad, es el sentido de pertenencia: enaltecerlo por medio de la adquisición y uso de estos artículos.

Otro aspecto importante, sobre el cual se podría profundizar en estudios posteriores, aborda la trascendencia de los artículos ofrecidos desde el punto de vista como souvenir, como una pequeña muestra de tradición que representa una comunidad. El cómo este tipo de artículos pueden, mediante su diseño y elaboración, expresar características representativas que identifiquen a la institución y logren generar reconocimiento por parte de sus integrantes y más aún de ajenos a la Universidad Nacional de Colombia -sede Medellín-, que al tener un artículo en sus manos salido de la tienda UN, puedan identificar claramente su procedencia.

\section{REFERENCIAS}

Aspara, J. (2009). Stock ownership as a motivation of brand-loyal and brand-supportive behaviors. Journal of marketing research, 427-436. 
Casas-Anguita, J.; Repullo-Labrador, J. R. Y Donado-Campos, J. (2003). La encuesta como técnica de investigación. Elaboración de cuestionarios y tratamiento estadístico de los datos (I). Atención primaria, 31(8), 527-538.

Dick, A. S. y Basu, K. (1994). Customer Loyalty: Toward an Integrated Conceptual Framework. Journal of the Academy of Marketing Science, 99-113.

Echauri, A. M. F.; Minami, H. y Sandoval, M. J. I. (2014). La Escala de Likert en la evaluación docente: acercamiento a sus características y principios metodológicos. Perspectivas Docentes, (50).

Fundación Universitaria del Área Andina. (2011). Creatividad e innovación como base para construir un país. Bogotá. Recuperado de

http://educacion.edu.co/contenidos/portal/ documentos/CID/Memorias-del-IIIEncuentro-Interinstitucional-de-Semillerosde-Investigacion-Sept-2011. pdf\#page=33

Kwon, H. y Armstrong, K. L. (2006). Impulse Purchases of Sport Team Licensed Merchandise: What Matters? Journal of Sport Management, 20,101-119.

Namakforoosh, M. N. (2000). Metodología de la investigación. Editorial Limusa.

Pelaez, A. y Macía, J. P. (2010). Influencia de la edad en la lealtad por marcas y productos de los consumidores de la ciudad de Medellín. Soluciones de Postgrado EIA, 5573.

Petit, C. \&y Charpentier, L. (2013). Identidad cultural en el objeto y diseño de souvenir El caso de Chiloé (tesis doctoral no publicada). Universidad Complutense de Madrid.
Recuperado de: http://eprints.ucm.es/22913/1/T34757.pdf

Phillips, J.; Roundtree, R. y Kim, D. (2014). Mind, body, or spirit? An exploration of customer motivations to purchase university licensed merchandise. Sport, Business and Management: An International Journal, 4(1), 71-87. doi:10.1108/SBM-08-20110069.

Rubio, N.; Villaseñor, N. y Oubiña, J. (2014). Consumer identification with store brands: Differences between consumers according to their brand loyalty. BRQ Business Research Quarterly. doi:10.1016/j.brq.2014.03.004

Salman, M.; Khan, S. y Gul, M. S. A. (2014). Factors Influencing Impulse Buying of Sports Team Merchandise in Developing Country: An Empirical Investigation. Recuperado de http://www.jespk.net/publications/169.pdf

Serrano, F. I y Goyeneche, D. (2008). Creación de una tienda universitaria con productos marca UIS. Recuperado de Repositorio UIS: http://repositorio.uis.edu.co/jspui/bitstrea m/123456789/5025/2/128821.pdf

Souvenirs. El caso de Chiloé (Tesis doctoral). Universidad complutense de Madrid, Madrid, España.

Universidad de Caldas (2014). Tienda Universitaria. Recuperado de http://www.ucaldas.edu.co/tienda/index.ht $\mathrm{ml}$

Universidad Pontificia Bolivariana (2014). Tienda Universitaria UPB. Recuperado de http://www.upb.edu.co/portal/page?_pagei $d=1054,28641639 \&$ dad=portal\&_schema= PORTAL 